\title{
Multifaceted Intervention Including Motivational Interviewing to Support Medication Adherence after Stroke/Transient Ischemic Attack: A Randomized Trial
}

\author{
Ulla Hedegaard ${ }^{a, c} \quad$ Lene Juel Kjeldsen $^{d} \quad$ Anton Pottegård $^{a}$ Søren Bak ${ }^{b}$ \\ Jesper Hallas ${ }^{a}$ \\ ${ }^{a}$ Clinical Pharmacology and Pharmacy, Institute of Public Health, University of Southern \\ Denmark, and ${ }^{b}$ Department of Neurology, and ${ }^{d}$ Hospital Pharmacy of Funen, Odense \\ University Hospital, Odense, and d Danish Research Unit for Hospital Pharmacy, Amgros I/S, \\ Copenhagen, Denmark
}

\section{Key Words}

Medication adherence $\cdot$ Motivational interviewing $\cdot$ Pharmacy services, hospital $\cdot$ Stroke $\cdot$

Ischemic attack, transient

\section{Abstract}

Background and Purpose: Adherence to medication is often suboptimal after stroke and transient ischemic attack (TIA), which increases the risk of recurrent stroke and death. Complex interventions and motivational interviewing (MI) have been proven effective in other areas of medicine. The objective of this study was to investigate the effectiveness of a multifaceted intervention including MI in improving medication adherence for secondary stroke prevention. Methods: In this randomized controlled trial, TIA and stroke patients receiving a pharmacist intervention in a hospital setting were compared with patients receiving usual care. The intervention consisted of a focused medication review, an MI-approached consultation and 3 follow-up telephone calls and lasted for 6 months. The primary outcome was a composite medication possession ratio (MPR) for antiplatelets, anticoagulants and statins in the year after hospitalization, assessed by analyzing pharmacy records and reported as both a continuous rate and a binary outcome. Secondary outcomes included composite MPRs at 3, 6 and 9 months as well as adherence and persistence to specific thrombopreventive medications at 12 months. Clinical outcomes included a combined end point of cardiovascular death, stroke or acute myocardial infarction. Patient satisfaction with the service was assessed

This study was conducted at Odense University Hospital, Odense, Denmark. ClinicalTrials.gov identifier: NCT01684176. 
Hedegaard et al.: Multifaceted Intervention Including Motivational Interviewing to Support Medication Adherence after Stroke/TIA: A Randomized Trial

for the intervention patients. Results: The analyses included 102 intervention patients and 101 controls. At 12 months, the median MPRs (IQR) were 0.95 (0.77-1) in the intervention group and $0.91(0.83-0.99)$ in the control group, and 28 and $21 \%$ of the patients, respectively, were nonadherent (MPR <0.80; risk difference: $7 \%$; $95 \%$ CI: -5 to $19 \%$ ). In both groups, the median MPR decreased over time. From 3 to 12 months, the MPR fell by $5 \%(p<0.05)$ in the intervention group and by $9 \%(p<0.05)$ in the control group, but between the groups, comparisons showed no statistically significant difference. No significant differences were found for adherence and persistence to specific thrombopreventive agents or for the clinical outcome. The intervention patients were satisfied with the service; about half of them reported increased knowledge about medication, and one third reported increased confidence with medication use. Pharmacists identified drug-related problems in one third of the patients. Conclusions: A multifaceted pharmacist intervention including MI did not improve adherence or persistence to secondary stroke prevention therapy and had no impact on clinical outcomes. However, due to the high adherence rates, only little room for improvement existed. Future studies should focus on patients at high risk of nonadherence and include outcomes more sensitive to the impact of behavioral interventions.

(C) 2014 S. Karger AG, Basel

\section{Introduction}

Adherence to preventive medication is often suboptimal after stroke and transient ischemic attack (TIA) [1-3]. Poor adherence and nonpersistence (discontinuation) to secondary prevention lead to poor health outcomes and substantial public health costs $[2,4]$. Targeting adherence may therefore be a key factor in reducing the incidence of recurrent stroke and vascular events.

Despite the obvious potential in improving medication adherence, a gold standard for interventions remains elusive. Nonadherence behavior occurs for a variety of reasons $[5,6]$. A Cochrane review found that complex interventions including a combination of several approaches (e.g. simplification of dose regimens, information, reminders, self-monitoring, counseling and telephone follow-ups) are more effective than simple interventions such as information-giving alone, but even the most effective complex interventions do not lead to great improvements [7].

Due to the marginal effect and poor efficacy of adherence interventions, novel cognitionbased techniques of behavior change have emerged. Among these, motivational interviewing (MI) is the most widely recognized [8]. MI is a counseling approach with emphasis on the patients' perspective and situation and facilitates behavior change by resolving their ambivalence and enhancing their empowerment. In recent years, MI has been proven effective in improving long-term medication adherence [8].

We performed a randomized trial to evaluate whether a pharmacist-led complex intervention including a focused medication review, an MI-approached consultation and follow-up telephone calls would improve medication adherence and persistence after stroke/TIA.

\section{Methods}

In this randomized controlled trial, TIA and stroke patients receiving a clinical pharmacist intervention in a hospital setting were compared with patients receiving usual (nonpharmacist) care. Their adherence and persistence to medication were measured by analyzing pharmacy records. 


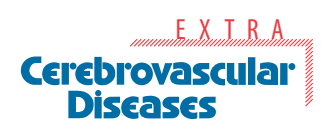

\begin{tabular}{l|l}
\hline \multicolumn{2}{l}{ Cerebrovasc Dis Extra 2014;4:221-234 } \\
\hline DOI: $10.1159 / 000369380$ & $\begin{array}{l}\text { C) 2014 S. Karger AG, Basel } \\
\text { www.karger.com/cee }\end{array}$ \\
\hline
\end{tabular}

Hedegaard et al.: Multifaceted Intervention Including Motivational Interviewing to

Support Medication Adherence after Stroke/TIA: A Randomized Trial

\section{Study Design and Participants}

The study was conducted at Odense University Hospital, Odense, Denmark. Patients with acute first-time ischemic stroke or TIA within the previous 30 days were included from the emergency ward or from 5 locations at the Department of Neurology ( 2 wards, 1 patient hotel, 1 rehabilitation center and 1 TIA outpatient clinic). Patients were eligible if they were $\geq 18$ years old, if they were prescribed at least 1 antiplatelet or anticoagulant medication and if they or cohabiting relatives dispensed the patients' medication. Patients were excluded if they lived in a care home or institution, if they received dose-dispensed medicine from a pharmacy, if medicine was dispensed by a home nurse or if they had a terminal illness or cognitive or physical impairment that would preclude the patient interview.

\section{Clinical Pharmacist Intervention}

Four clinical pharmacists employed at the hospital pharmacy were trained in providing the intervention. The intervention consisted of 3 elements: (1) a focused medication review, (2) a patient interview and (3) 3 follow-up telephone calls to the patient within the first 6 months after discharge. The medication review focused on thrombopreventive agents and potential adherence problems related to these. Advice on relevant drug-related problems (DRPs) was provided to the physician in charge. The medication review was followed by a 30-min face-to-face patient interview to support adherence and lifestyle changes. The dialogue was based on the concept of MI [9]. At the end of the interview, the patients received a written summary of the interview including their own goals and a list of jointly agreed-on actions that should be taken. The patients were contacted by telephone 1 week as well as 2 and 6 months after discharge. After the second and third interviews, the patients were mailed a written summary including shared goals and plans. During the third follow-up, patient satisfaction and experiences with the intervention were explored. The intervention and pharmacist training are described in detail in the supplementary material (for all online suppl. material, see www.karger.com/doi/10.1159/000369380).

\section{Usual Care Group}

Patients in the control group received standard care without the clinical pharmacist intervention. Standard care was performed by physicians and included diagnosing, initiation of thrombopreventive treatment and short counseling about lifestyle (diet, exercise, smoking and drinking). Follow-up visits to the outpatient clinic did not occur on a regular basis, only in case of special problems. Two months after the study was initiated, a secondary prevention outpatient clinic run by nurses was established. The clinic offered stroke and TIA patients a consultation 14 days after the primary contact and stroke patients an additional visit 3 months after discharge. At the clinic, a broad range of risk factors and conditions including medication adherence and lifestyle behavior were addressed.

\section{Baseline Data and Process Outcomes}

Baseline data on age, sex, diagnosis, hospital setting, severity of stroke and cerebrovascular risk factors and prescribed medications were collected from the patients' electronic medical records (EMRs). For patients treated with antithrombotics, statins or antihypertensives before entering the study, a baseline overall adherence to thrombopreventive agents was calculated from refill data using an observation period of 9 months prior to study entry. Baseline adherence was calculated similarly to the main outcomes as described below, except that baseline adherence was not adjusted for prescribing changes and hospitalization. Baseline adherence was assessed after the intervention had been completed. DRPs were divided into 7 categories using an adapted version of the classification described by Strand et al. [10] together with suggested actions. A DRP was defined as an undesirable patient expe- 
Hedegaard et al:: Multifaceted Intervention Including Motivational Interviewing to Support Medication Adherence after Stroke/TIA: A Randomized Trial

rience involving drug therapy that actually or potentially interfered with the desired patient outcome [10]. Information about physicians' acceptance of the recommendations was collected from the patients' EMRs. The time spent on the intervention was recorded.

\section{Outcomes and Assessments}

Adherence and persistence measures were estimated for antiplatelets, anticoagulants, statins and antihypertensives from data obtained from the Odense University Pharmacoepidemiological Database (OPED) [11]. The register covers all prescriptions for reimbursed medicine redeemed at Danish pharmacies by inhabitants of the Region of Southern Denmark.

\section{Primary Outcome}

The primary outcome was overall adherence to the thrombopreventive regimen, i.e. antiplatelets, anticoagulants and statins, in the year after hospitalization, reported as both a continuous and a binary outcome. Adherence to vitamin $\mathrm{K}$ antagonists was not included, because medication data were less reliable due to frequent dose titration.

The adherence assessment was based on the medication possession ratio (MPR) measure [12] defined as the amount of drug available from refills during follow-up relative to the amount prescribed. The MPR was capped at 1.0 as rates exceeding 1.0 were regarded as full adherence rather than overmedication [13]. The MPR estimate was refined in two ways as described by Vollmer et al. [14]: (1) expanding the denominator to the entire follow-up period (contrary to the period between the first and last dispensing) and (2) accounting for medication that individuals possessed at the start of the follow-up period. The calculation was further refined by adjusting for hospitalization days and accounting for prescribing change (dose, discontinuation or initiation) during the follow-up period. Information on changes was obtained from the EMR, the OPED and the Shared Medication Record, the last of which hosts information on active medication and prescriptions for all Danish citizens [15].

The continuous outcome, composite MPR, was a time-weighted mean of MPRs for antiplatelets, anticoagulants and statins, where the MPR for each drug was weighted by the duration of its observation as described by Steiner et al. [16]. The binary outcome was the number of nonadherent (composite MPR <0.80) and adherent (composite MPR $\geq 0.80$ ) patients in the year of follow-up. We considered a patient adherent/persistent if he switched medication within a class. Switching from antiplatelets to anticoagulants was also accepted, as cardioembolic reasons for stroke were often only conclusively diagnosed after discharge.

Patients were followed up until one of the following events first occurred: drugs discontinued by a hospital physician; death; dose-dispensed medicine; medicine dispensed by a home nurse; institutionalization; emigration from the Region of Southern Denmark, or the end of the study period.

\section{Secondary Outcomes}

The secondary outcomes were overall adherence to antiplatelets, anticoagulants and statins at 3, 6 and 9 months as well as adherence to low-dose acetylsalicylic acid (ASA), dipyridamole, clopidogrel, statins and antihypertensive agents, all at 12 months.

Persistence measures were also secondary outcomes and were calculated for clopidogrel, ASA, dipyridamole and statins. Nonpersistence was defined at the first episode of failing to redeem a prescription within 90 days after the last date covered by the preceding prescription [12]. The days to discontinuation were the number of days from discharge to the day for which the final fill provided dosing [12], and they are visually displayed by Kaplan-Meier curves. Persistence was also reported as a dichotomous variable measured at the end of the observation period, i.e. persistent or nonpersistent. A sensitivity analysis was undertaken to investigate the influence of using grace periods of $60,120,150$ and 180 days instead of the 
90 days used in the main analysis. Acceptance of treatment was defined as redeeming a prescription within 30 days after discharge and was estimated for medication initiated in hospital.

As a secondary clinical outcome, a combined end point of death, acute myocardial infarction (AMI) or hemorrhagic or ischemic stroke was reported. Data on hospital readmission to a medical department due to stroke and AMI were obtained from the Danish National eHealth Portal, which holds information on all Danish public hospital admissions. Mortality data were obtained from EMRs and the Danish Civil Registration system.

\section{Randomization, Blinding and Approvals}

The randomization process was performed by the clinical trial group at the hospital pharmacy. A total of 200 patients were allocated using a 1:1 allocation ratio. Randomization block sizes of 4 and 6 were generated by a computerized random number generator prior to enrollment, and the allocation was concealed in numbered opaque envelopes. To ensure sufficient power in the two groups, a randomization sequence of an additional 11 patients was generated late in the inclusion period as substitutions for those patients who deviated from the protocol before discharge (fig. 1).

All participants gave informed consent, and the study protocol was approved by the Regional Scientific Ethics Committees for Southern Denmark and the Danish Registry Board.

\section{Sample Size}

The sample size calculation was based on an assumed nonadherence rate of about $50 \%$ [4] and an absolute risk reduction of about $20 \%$ as seen in pharmacist-led interventions in hypertensive patients [17]. To detect a $20 \%$ absolute risk reduction with a power of $80 \%$, a significance level at 5\% and an anticipated dropout rate of $10 \%$, a total sample size of 100 patients in each subgroup was, initially, considered appropriate.

\section{Statistical Analysis}

All data collected for assessment of adherence were entered by 2 different persons into 2 separate data files and compared and corrected using EpiData version 3.1. The data were analyzed using Stata version 13. The researcher and research assistant were kept blinded to the allocation when collecting data from the follow-up period, and the researcher was kept blinded when assessing and analyzing outcomes.

Results are reported as numbers and percentages for categorical variables and means (SD) or medians (IQR) for continuous variables. Binary adherence estimates are given as risk differences (RDs) and 95\% CIs. Adherence over time was compared using a random effect logistic regression model. Adherence between the groups was compared with the WilcoxonMann-Whitney two-sided test. Binary outcomes were compared using Fisher's exact test. Persistence over time was compared using survival statistics with the Cox proportional hazard model. Clinical outcomes are reported as relative risk ratios and 95\% CIs.

The primary analyses were performed for all patients with applicable medication data according to the protocol. We conducted an exploratory per-protocol analysis.

\section{Results}

Participants and Recruitment

Patients were recruited from August 2012 to March 2013 and followed up until 1 year after discharge. Figure 1 shows the patient flow through the study. Of 243 patients invited to participate, $32(13 \%)$ declined. Of the 211 randomized patients, 8 were excluded from the 
Hedegaard et al.: Multifaceted Intervention Including Motivational Interviewing to Support Medication Adherence after Stroke/TIA: A Randomized Trial

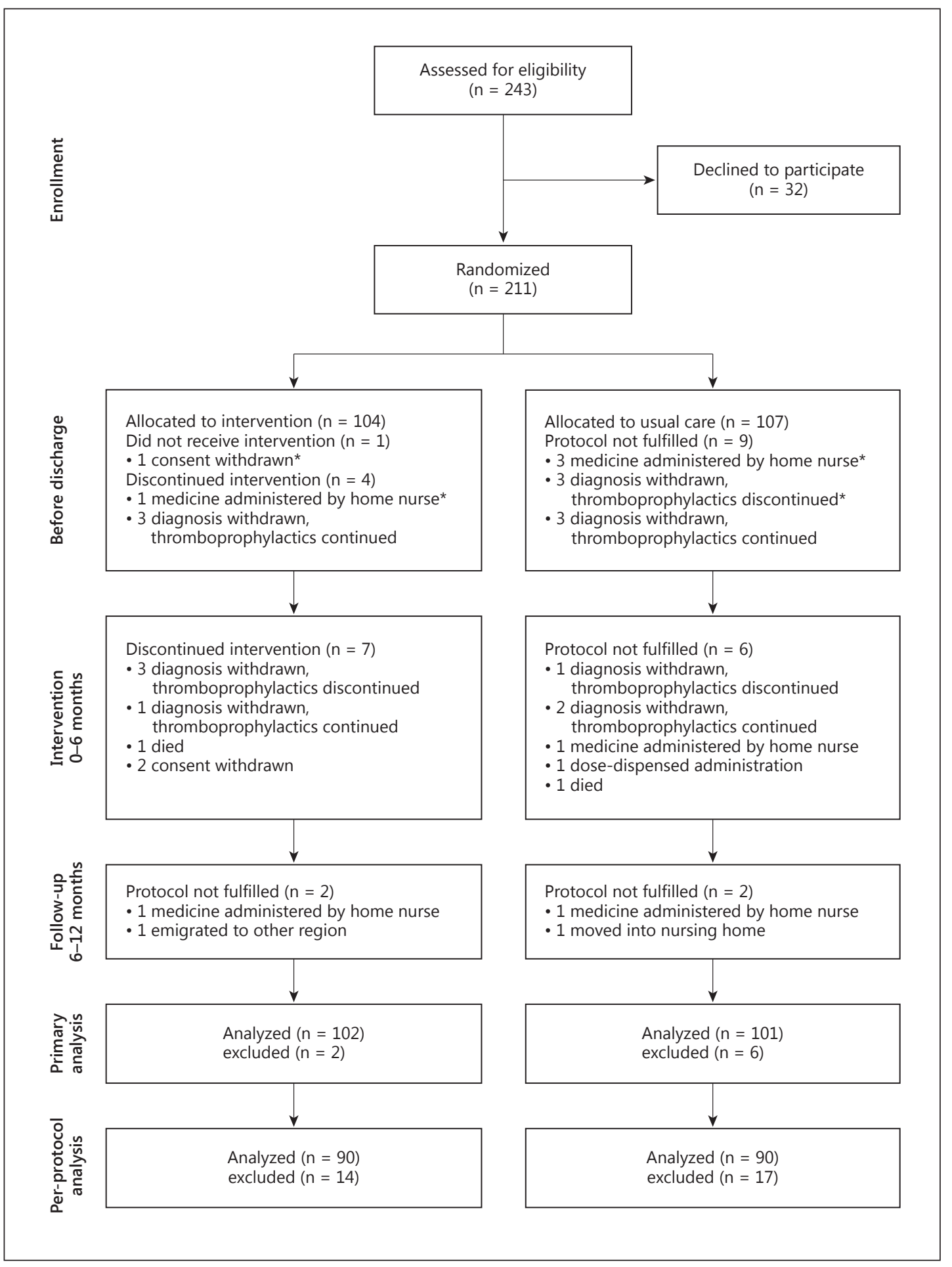

Fig. 1. Participant flow diagram. * Patients excluded from primary analysis. 
primary analyses (fig. 1) as adherence could not be estimated according to the protocol. This left 203 evaluable patients. A further 3 patients were excluded from the primary end point analysis: 2 patients had discontinued antithrombotics before discharge and 1 patient had received only warfarin (adherence not assessed due to frequent dose titration). All 3 received antihypertensive agents, which are included in the secondary end points. The most frequent violation against the protocol was revision of the stroke diagnosis $(n=16)$, followed by dose dispensed by a home nurse $(n=7)$. Details on the violations are shown in figure 1 . In total, 31 patients had significant protocol violations, and 180 patients remained for the per-protocol analysis.

\section{Baseline Data}

The baseline demographics and clinical characteristics are shown in table 1 . The median age of the patients was 67 years, and $61 \%$ were male. Diagnoses were equally divided between ischemic stroke and TIA, and the Scandinavian Stroke Scale scores were at the upper level, indicating that most patients were only slightly affected by apoplectic symptoms. In line with this, the number of patients with atrial fibrillation was low, as atrial fibrillation is associated with more severe strokes [18]. An equal number of patients in the intervention and the usual care group (52.0 vs. $53.5 \%$ ) visited the secondary prevention clinic established during the study. The groups were well balanced, except in three respects: more patients in the intervention group were smokers ( 38.2 vs. $26.7 \%$ ) and had heart valve disease ( 6.9 vs. $0 \%$ ), and the proportion of nonadherent patients at baseline tended to be lower in the intervention group (41 vs. 52\%).

\section{Intervention}

In total, 103 patients completed the medication review and the interview. The first, second and third follow-up telephone calls were completed by 97, 94 and 92 patients, respectively. The main reason why 11 patients were excluded from finishing the intervention was diagnosis revision (fig. 1). For the 92 patients who had completed the intervention, the mean total time the pharmacists spent on each patient was $2 \mathrm{~h} 15 \mathrm{~min}$ (SD $27 \mathrm{~min}$ ).

The focused medication review revealed 35 DRPs among 32 patients. The most common DRP identified was need for additional drug therapy $(n=15)$ and addressed the lack of prescription of statin therapy $(n=12)$, antithrombotics $(n=2)$ and antihypertensives $(n=1)$ despite clear indications. The remaining DRPs were reconciliation errors $(n=7)$, adverse drug reaction $(n=4)$, inappropriate dose $(n=3)$, inappropriate drug $(n=3)$, drug interactions $(\mathrm{n}=2)$ and no medical indication $(\mathrm{n}=1)$. The agents most commonly involved were statins $(\mathrm{n}=18)$ and ASA $(\mathrm{n}=6)$.

The most frequent recommendations to the physicians were initiation of drug therapy $(n=19)$, resolving reconciliation errors $(n=7)$ and change of drug $(n=3)$. The physicians accepted $27(79 \%)$ of the recommendations; 3 (9\%) were refused, and to $5(14 \%)$ they gave no response.

After discharge, a change to the simpler clopidogrel regimen instead of dipyridamole and ASA frequently occurred. The number of patients treated with clopidogrel increased by $74 \%$ (from 23 to 40) in the intervention group and by 55\% (from 20 to 31) in the control group (relative risk ratio: 1.53 ; 95\% $\mathrm{CI}$ : $0.75-3.10$ ).

\section{Medication Adherence and Persistence}

For the primary end point, i.e. the composite MPR over 1 year, no significant differences were seen between the intervention and the control group in the continuous or binary outcome (table 2). In both groups, the median adherence rate (IQR) was high, i.e. $0.95(0.77-1)$ and 0.91(0.83-0.99) in the intervention and the control group, respectively. 
Table 1. Baseline demographics and clinical characteristics

\begin{tabular}{|c|c|c|}
\hline Variable & $\begin{array}{l}\text { Intervention group } \\
(\mathrm{n}=102)\end{array}$ & $\begin{array}{l}\text { Control group } \\
(n=101)\end{array}$ \\
\hline Men & $61(59.8)$ & $63(62.4)$ \\
\hline Age, years & $64(56-73)$ & $68(61-73)$ \\
\hline \multicolumn{3}{|l|}{ Stroke characteristics } \\
\hline Ischemic stroke & $53(52.0)$ & $50(49.5)$ \\
\hline TIA & $47(46.1)$ & $49(48.5)$ \\
\hline Stroke/TIA diagnosis withdrawn & $2(2.0)$ & $2(2.0)$ \\
\hline Scandinavian Stroke Scale score ${ }^{\mathrm{a}}$ & $58(54-58)$ & $58(55-58)$ \\
\hline \multicolumn{3}{|l|}{ Current hospital contact } \\
\hline Acute ward & $14(13.7)$ & $13(12.9)$ \\
\hline Neurological ward & $64(62.7)$ & $70(69.3)$ \\
\hline Neurological rehabilitation ward & $5(4.9)$ & $6(5.9)$ \\
\hline Neurological outpatient clinic & $19(18.6)$ & $12(11.9)$ \\
\hline Length of hospital stay, days & $2(1-5)$ & $3(1-6)$ \\
\hline Neurological secondary prevention clinic & $53(52.0)$ & $54(53.5)$ \\
\hline \multicolumn{3}{|l|}{ Thrombopreventive medications at discharge } \\
\hline Antiplatelets & $96(94.1)$ & $97(96.0)$ \\
\hline ASA + dipyridamole & $69(67.6)$ & $73(72.3)$ \\
\hline Clopidogrel & $23(22.5)$ & $20(19.8)$ \\
\hline Anticoagulants & $6(5.9)$ & $5(5.0)$ \\
\hline Statins & $82(80.4)$ & $75(74.3)$ \\
\hline Antihypertensive agents & $56(54.9)$ & $50(49.5)$ \\
\hline \multicolumn{3}{|l|}{ Number of medications } \\
\hline Thrombopreventive agents & $3(3-4)$ & $3(3-4)$ \\
\hline Thrombopreventive agents initiated at current contact & $2(1-3)$ & $2(2-3)$ \\
\hline Total number of unique medications & $5(3-7)$ & $4(3-6)$ \\
\hline \multicolumn{3}{|l|}{ Baseline adherence to thrombopreventive medications ${ }^{b}$} \\
\hline Composite MPR & $0.89(0.67-0.98)$ & $0.79(0.49-0.97)$ \\
\hline Nonadherent (composite MPR $<0.8$ ) & $24(40.7)$ & $25(52.1)$ \\
\hline \multicolumn{3}{|l|}{ Risk factors } \\
\hline Hypertension & $55(53.9)$ & $46(45.5)$ \\
\hline Atrial fibrillation & $7(6.9)$ & $10(9.9)$ \\
\hline Diabetes mellitus & $8(7.8)$ & 7 (6.9) \\
\hline Dyslipidemia & $85(83.3)$ & $76(75.2)$ \\
\hline Ischemic heart disease & $9(8.8)$ & $12(11.9)$ \\
\hline Claudicatio intermittens & $4(3.9)$ & $0(0.0)$ \\
\hline Congestive heart failure & $2(2.0)$ & $1(1.0)$ \\
\hline Heart valve disease & 7 (6.9) & $0(0.0)$ \\
\hline High alcohol consumption ${ }^{\mathrm{c}}$ & $10(9.8)$ & $11(10.9)$ \\
\hline Current smoking & $39(38.2)$ & $27(26.7)$ \\
\hline $\mathrm{BMI}>30$ & $18(17.6)$ & $15(14.9)$ \\
\hline
\end{tabular}

Values denote $\mathrm{n}(\%)$ or medians (IQR). ${ }^{\mathrm{a}}$ Included in the Scandinavian Stroke Scale analysis: intervention group, $n=70$; control group, $n=63 .{ }^{b}$ Mean MPR for all thrombopreventive agents for a 270-day period ahead of the inclusion. Included in the MPR analysis: intervention group, $\mathrm{n}=59$; control group, $\mathrm{n}=48$. ${ }^{\mathrm{c}}$ Weekly consumption: women $>14$ drinks, men $>21$ drinks.

At 3, 6 and 9 months, the composite MPRs were high at all time points, with median values above 0.90. Between-group comparisons showed no significant differences (fig. 2). Within-group comparisons showed a small, statistically significant impact of time on adherence rate in both groups. From 3 to 12 months, the median MPR fell from 1 to 0.95 ( $p<$ $0.05)$ in the intervention group and from 1 to $0.91(\mathrm{p}<0.05)$ in the control group, showing a small nonsignificant trend towards a larger fall in the control group. 
Table 2. Adherence to thrombopreventive medications

\begin{tabular}{|c|c|c|c|c|}
\hline Variable & $\begin{array}{l}\text { Intervention } \\
\text { group }\end{array}$ & $\begin{array}{l}\text { Control } \\
\text { group }\end{array}$ & $\mathrm{RD}(95 \% \mathrm{CI})$ & $\mathrm{p}$ value \\
\hline Primary end point, $n$ & 100 & 100 & & \\
\hline Composite MPR ${ }^{\mathrm{a}}$ & $0.95(0.77-1)$ & $0.91(0.83-0.99)$ & NA & 0.85 \\
\hline Nonadherent (composite MPR <0.8) & $28(28)$ & $21(21)$ & $7(-5$ to 19$)$ & 0.32 \\
\hline ASA, $n$ & 76 & 86 & & \\
\hline MPR & $1(0.77-1)$ & $1(0.71-1)$ & NA & 0.25 \\
\hline Nonadherent (MPR <0.8) & $20(26)$ & $24(28)$ & $-2(-15$ to 12$)$ & 0.86 \\
\hline Dipyridamole, $\mathrm{n}$ & 67 & 74 & & \\
\hline MPR & $0.99(0.80-1)$ & $0.99(0.83-1)$ & NA & 0.91 \\
\hline Nonadherent (MPR <0.8) & $17(25)$ & $16(22)$ & $4(-10$ to 18$)$ & 0.69 \\
\hline Clopidogrel, $\mathrm{n}$ & 46 & 37 & & \\
\hline MPR & $1(0.91-1)$ & $1(0.97-1)$ & NA & 0.21 \\
\hline Nonadherent (MPR <0.8) & $6(13)$ & $2(5)$ & $8(-5$ to 20$)$ & 0.29 \\
\hline Statins, $\mathrm{n}$ & 90 & 87 & & \\
\hline MPR & $1(0.83-1)$ & $0.98(0.82-1)$ & NA & 0.68 \\
\hline Nonadherent (MPR <0.8) & $20(22)$ & $18(21)$ & $2(-11$ to 14$)$ & 0.86 \\
\hline Antihypertensive agents, $\mathrm{n}$ & 72 & 63 & & \\
\hline Composite MPR ${ }^{\mathrm{b}}$ & $0.98(0.83-1)$ & $0.96(0.78-1)$ & NA & 0.74 \\
\hline Nonadherent (composite MPR <0.8) & $16(22)$ & $16(25)$ & $-3(-18$ to 11$)$ & 0.69 \\
\hline
\end{tabular}

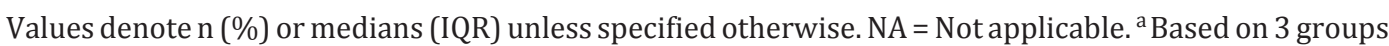
of medications: antiplatelets, anticoagulants and statins. ${ }^{b}$ Based on moxonidine, diuretics, calcium antagonists, renin-angiotensin agents and beta-blockers.

Fig. 2. Boxplot of overall adherence (composite MPR) at baseline as well as at 3, 6, 9 and 12 months. The composite MPR is based on 3 groups of medications: antiplatelets, anticoagulants and statins. The box displays the IQR and the median. The whiskers display 1.5 IQR. Outliers are excluded. At baseline: $\mathrm{n}=107$; median number of preventive drugs per patient = 1. At 3, 6, 9 and 12 months: $\mathrm{n}=$ 200 ; median number of preventive drugs $=3$.

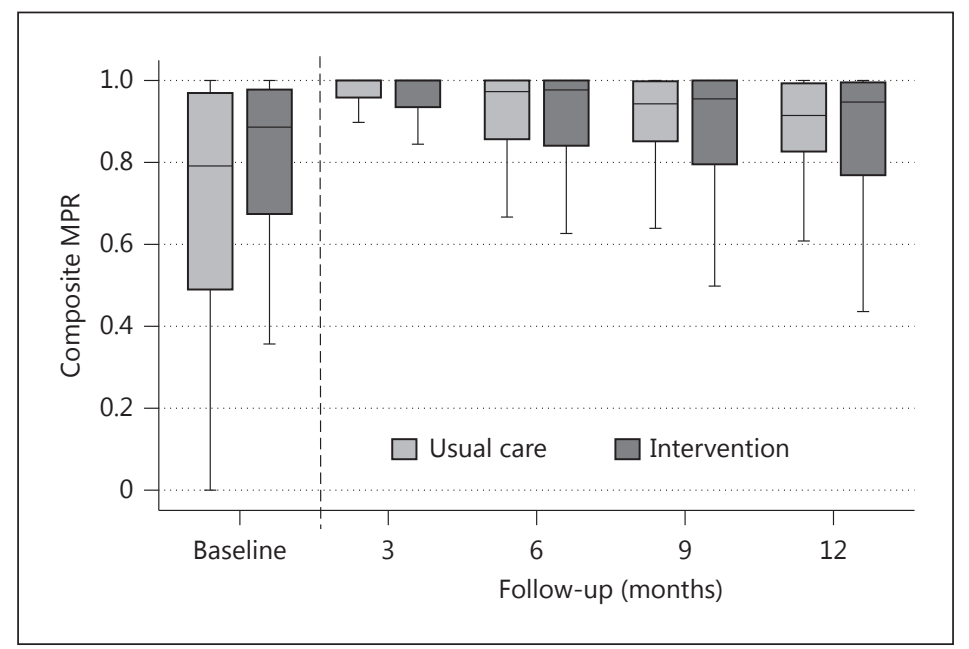

The difference between baseline adherence and adherence after the stroke event was not analyzed formally, as the methods for measuring adherence differ. Furthermore, baseline adherence measures are also more imprecise due to fewer patients and fewer drugs. However, the figures indicated a marked increase in adherence following the stroke event (fig. 2) for both the intervention and the control group.

Separate analyses of adherence at 12 months for statins, antihypertensive agents and antiplatelets did not reveal statistically significant differences between the intervention and 


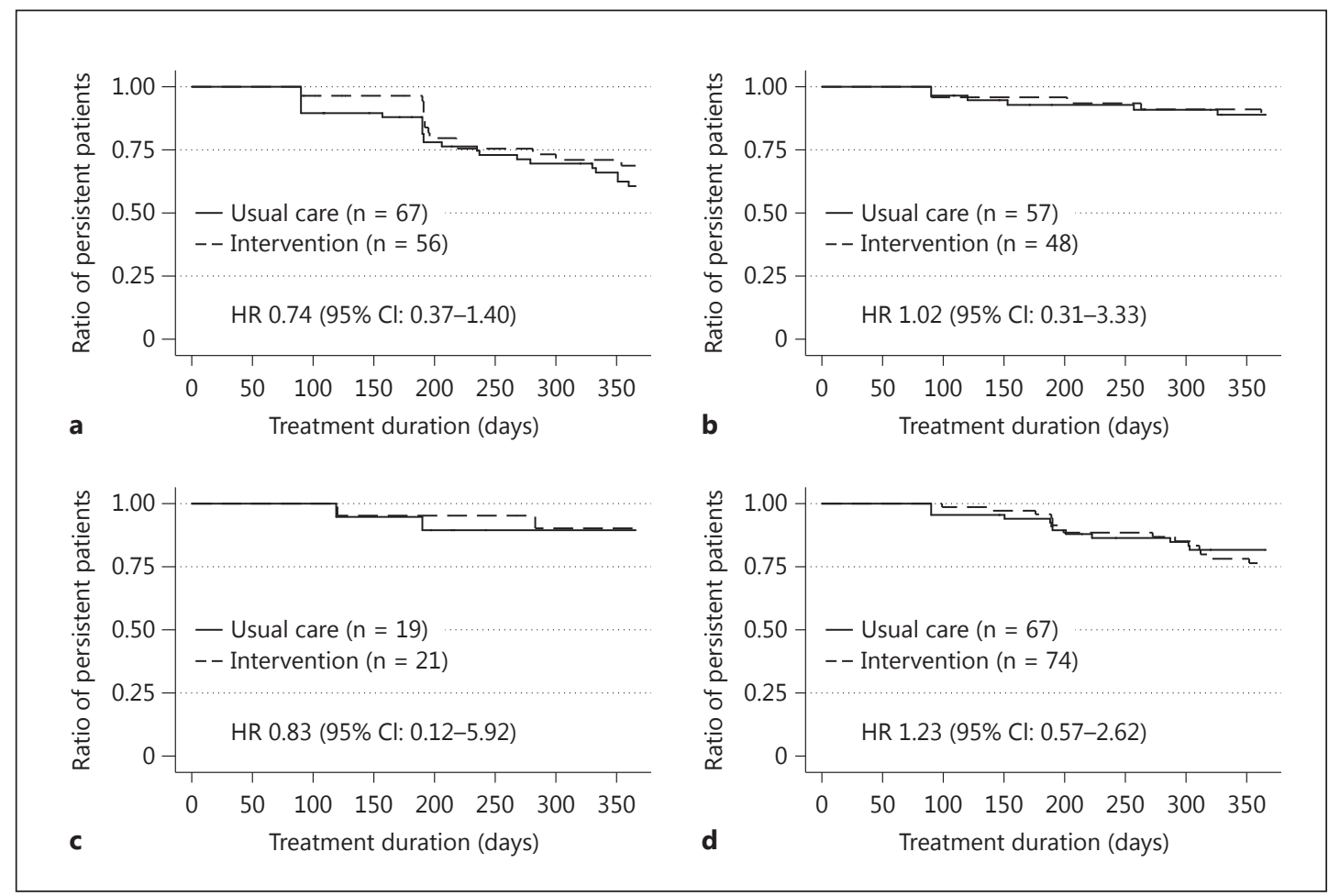

Fig. 3. Kaplan-Meier plots of time to nonpersistence for ASA (a), dipyridamole (b), clopidogrel (c) and statins (d). HR = Hazard ratio.

the control group (table 2). Per-protocol analyses of adherence at 12 months did not markedly change the results from the primary analyses (online suppl. table I).

Nonpersistence was not significantly prevented by the intervention for any of the specific drug classes analyzed (fig. 3). Of note, the highest rate of nonpersistence occurred with ASA. At 12 months, 27\% (15/56) of the patients in the intervention group were nonpersistent compared to $36 \%$ (24/67) of the patients in the control group (RD: $-9 \%$; $95 \% \mathrm{CI}:-25$ to $7 \%$ ). For dipyridamole, clopidogrel and statins, the proportion of nonpersistent patients in the intervention group was similar to that in the control group, i.e. 10 versus $11 \%, 10$ versus $11 \%$ and 20 versus $18 \%$, respectively. The sensitivity analysis for grace periods of 30 , 60,120 and 150 days showed increasing persistence with an increasing length of the grace period, but the overall result of no differences between groups was preserved (results not shown).

Nonacceptance was low. Of the 186 and 206 drug prescriptions during hospitalization in the intervention and the control group, respectively, only 1 prescription in each group was not redeemed within 30 days (RD: $0.05 \%$; $95 \% \mathrm{CI}:-1.4$ to $1.5 \%$ ).

\section{Clinical Outcome}

No significant differences were found for the composite clinical end point of death, stroke or AMI, which was reached by $5 \%(5 / 102)$ in the intervention group and $5 \%(5 / 101)$ in the control group (relative risk ratio: $0.99 ; 95 \% \mathrm{CI}$ : 0.30-3.32). In both groups, the distribution of the events was 1 AMI, 2 deaths and 2 strokes.

The intervention patients reported satisfaction with their participation in the study. A heightened focus on lifestyle change and increased knowledge about their medications were 
reported by about half the patients. Approximately one third of the patients had experienced more confidence with medication use, better skills for the correct use of medications and higher quality of life (online suppl. table II).

\section{Discussion}

Our study did not show significant differences in adherence, persistence or clinical outcomes between patients receiving an MI-based pharmacist intervention and patients receiving usual care. The most important explanation for the absence of an intervention effect is probably the surprisingly high adherence rate in both groups, thus leaving little room for improvement. Several factors may have contributed to this. Firstly, the prospect of having stroke relapse might induce a high level of adherence in some patients. For both groups, the stroke event itself seemed to improve adherence dramatically. Secondly, there might have been a carryover between groups, since intervention and control patients were recruited at the same units. For example, the clinical staff may have used some of the intervention elements on the control group. Thirdly, a secondary prevention clinic was established during our study, which may have improved adherence in both groups. Fourthly, the patients included usually had minor strokes and therefore probably fewer risk factors for nonadherence [3]. Fifthly, our definitions of persistence may have been too conservative. Except for low-dose ASA, the proportion of nonpersistent patients at 1 year was low compared to results from other studies based on refill data [1, 3]. These studies used a definition of persistence different from ours, e.g. shorter grace periods [3], and, as suggested by our sensitivity analyses, our long grace period might explain our low rate of nonpersistence. Finally, with a longer follow-up period, a difference may have developed as we observed a trend towards a smaller fall in the adherence rate in the intervention group compared to the control group. We find it unlikely that selection bias plays a major role in the high adherence rate, since only $13 \%$ of the patients declined participation. The patients were informed that data on their filled prescriptions would be collected. However, being aware of this data collection method is less likely to influence adherence as compared to, for instance, assessing adherence by pill count or electronic monitoring devices, where patients will be reminded of their participation in the study on a daily basis or on 'pill count days' $[19,20]$.

Our intervention included elements essential for any well-designed adherence intervention $[4,7]$. It was personalized and separately included multiple components proven effective in other settings $[7,8,17]$. Still, a possible explanation for the lack of an overall effect could lie in the nature of our intervention. However, due to the high adherence rates, the efficacy of the intervention could not be properly assessed. Settings with more room for improvement should be ensured in future studies.

The main strengths of our study are the use of an MI-based multicomponent intervention, objective adherence measures, an appropriate time frame of follow-up [7] and trained pharmacists to reduce bias and increase generalizability. Until now, 14 randomized intervention studies have assessed adherence after stroke and TIA [21,22], and all suffer methodological flaws due to small sample sizes, short follow-up periods or subjective (e.g. self-reported) adherence measures that tend to overestimate adherence [23]. Only one pilot study used an objective assessment (electronic recording of pill taking) together with self-reported adherence [24]. This study was also the only one reporting a significant difference in medication adherence between an intervention and a control group. Their intervention was cognition based, with 2 individualized sessions helping participants to establish a better medication taking routine and addressing barriers to implementation. Cognition-based techniques such as this one also include MI. According to a recent meta-analysis [8], these techniques improve the rate of doses taken by about $6 \%$. 


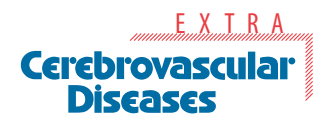

\begin{tabular}{l|l}
\hline \multicolumn{2}{l}{ Cerebrovasc Dis Extra 2014;4:221-234 } \\
\hline DOI: $10.1159 / 000369380$ & $\begin{array}{l}\text { C) 2014 S. Karger AG, Basel } \\
\text { www.karger.com/cee }\end{array}$ \\
\hline
\end{tabular}

Hedegaard et al.: Multifaceted Intervention Including Motivational Interviewing to Support Medication Adherence after Stroke/TIA: A Randomized Trial

To our knowledge, our study is the first randomized trial investigating the effect of MI on medication adherence after stroke, by using objective assessments of adherence. Only two underpowered studies using self-reported adherence have investigated the effect of MI in stroke patients, both with negative results $[25,26]$. Both interventions were complex and led by nurses. We shared the elements of MI and telephone calls, but our study also focused on pharmacists' optimization of drug therapy.

No effect was seen for the composite clinical end point. Given the fairly low event rate in both groups, this is hardly surprising. It has been discussed whether clinical events and mortality are sensitive and relevant parameters for an evaluation of clinical pharmacy services. Aspects of health-related quality from a patient's perspective may thus be more appropriate [27]. Our patient evaluation addressed some of these aspects and suggested that the intervention had an influence on patients' empowerment to manage their medication and even on their quality of life. This evaluation was only performed for the intervention group, which limits the values of the results. However, patient experience and medication selfefficacy should be explored with validated methods in future studies. Another potential secondary benefit is that pharmacists identified DRPs related to the patients' preventive therapy in one third of the subjects. Most suggestions were accepted by the physicians. Although not a predefined outcome, our intervention tended to promote a change to the simpler clopidogrel regimen after discharge.

There are some limitations to our study. Firstly, we used pharmacy refill data to assess adherence in contrast to clinical trials that have traditionally used pill counts [28]. Compared to other reliable objective methods such as pill counts and electronic monitoring devices, the refill method was the method that best matched our goals as it is feasible for analyzing multiregimen adherence and nonpersistence [19]. Also, it is inexpensive and less interfering and may therefore affect patient behavior to a lesser extent. Although refilling a prescription may result in stockpiling and does not unequivocally mean that the patient took the medication, the use of pharmacy refill data has been validated as a measure of adherence [16], and such data have been used in other interventions studies [28]. Furthermore, we were able to account for medication discontinuation, dose changes and days spent in hospital, which improved our estimate. Secondly, we had adherence as our primary outcome, and not clinical events such as mortality or stroke relapse. Thirdly, the pharmacists, patients and treating physicians could not be blinded to the allocation. This problem is largest for subjective outcomes, and our main outcomes were objectively assessed, using data from registries. The data collection and analysis during follow-up were blinded. Fourthly, we could not include those patients with most severe stroke as they might not have been capable of participating in our intervention (e.g. because of aphasia or cognitive impairments). Patients with more severe stroke might have a higher number of risk factors for nonadherence [3] and might potentially benefit more in terms of clinical outcomes.

\section{Conclusions}

An individualized pharmacist intervention for stroke and TIA patients did not improve adherence or persistence to secondary prevention therapy and had no impact on clinical outcomes. The adherence rate was high, which left little room for improvement and emphasizes that future studies should focus on targeting patients at high risk of nonadherence and include outcomes more sensitive to the impact of behavioral interventions. 


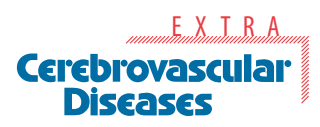

Cerebrovasc Dis Extra 2014;4:221-234

DOI: $10.1159 / 000369380$

Hedegaard et al.: Multifaceted Intervention Including Motivational Interviewing to

Support Medication Adherence after Stroke/TIA: A Randomized Trial

\section{Acknowledgements}

Lisbeth Muurholm, Head of the Hospital Pharmacy Funen, provided a perfect organizational base for performing the study, and we are indebted to her, the intervention pharmacists and the rest of the personnel involved. We would also like to thank the participating wards from Odense University Hospital. We thank Henrik Horneberg for careful proofreading.

The work was funded by grants from Odense University Hospital, the University of Southern Denmark, the hospital pharmacies and the Amgros I/S Research Development Foundation as well as from the Actavis Foundation.

\section{Disclosure Statement}

Anton Pottegård has participated in research projects funded by AstraZeneca, with grants paid to the institution where he was employed. Jesper Hallas has participated in research projects funded by Novartis, Pfizer, Merck Sharp \& Dohme and Nycomed, with grants paid to the institution where he was employed. He has personally received fees for teaching or consulting from the Danish Association of Pharmaceutical Manufacturers and from Nycomed, Pfizer, Novartis, AstraZeneca and Leo Pharmaceuticals. Ulla Hedegaard, Lene Juel Kjeldsen and Søren Bak report no disclosures.

\section{References}

1 Glader EL, Sjölander M, Eriksson M, Lundberg M: Persistent use of secondary preventive drugs declines rapidly during the first 2 years after stroke. Stroke 2010;41:397-401.

2 Weimar C, Cotton D, Sha N, Sacco RL, Bath PM, Weber R, Diener HC: Discontinuation of antiplatelet study medication and risk of recurrent stroke and cardiovascular events: results from the PRoFESS study. Cerebrovasc Dis 2013;35:538-543.

-3 Bushnell CD, Olson DM, Zhao X, Pan W, Zimmer LO, Goldstein LB, Alberts MJ, Fagan SC, Fonarow GC, Johnston SC, Kidwell C, Labresh KA, Ovbiagele B, Schwamm L, Peterson ED: Secondary preventive medication persistence and adherence 1 year after stroke. Neurology 2011;77:1182-1190.

4 Sabaté E: Adherence to long-term therapies: evidence for action. Geneva, World Health Organization, 2003.

5 Kardas P, Lewek P, Matyjaszczyk M: Determinants of patient adherence: a review of systematic reviews. Front Pharmacol 2013;4:91.

6 Horne R, Weinman J: Patients' beliefs about prescribed medicines and their role in adherence to treatment in chronic physical illness. J Psychosom Res 1999;47:555-567.

7 Haynes RB, Ackloo E, Sahota N, McDonald HP, Yao X: Interventions for enhancing medication adherence. Cochrane Database Syst Rev 2008;2:CD000011.

8 Easthall C, Song F, Bhattacharya D: A meta-analysis of cognitive-based behaviour change techniques as interventions to improve medication adherence. BMJ Open 2013;3:e002749.

-9 Rollnick S, Miller WR, Butler CC: Motivational Interviewing in Health Care: Helping Patients Change Behavior. New York, Guilford, 2008.

10 Strand LM, Morley PC, Cipolle RJ, Ramsey R, Lamsam GD: Drug-related problems: their structure and function. DICP 1990;24:1093-1097.

11 Gaist D, Sørensen HT, Hallas J: The Danish prescription registries. Dan Med Bull 1997;44:445-448.

12 Peterson AM, Nau DP, Cramer JA, Benner J, Gwadry-Sridhar F, Nichol M: A checklist for medication compliance and persistence studies using retrospective databases. Value Health 2007;10:3-12.

13 Halpern MT, Khan ZM, Schmier JK, Burnier M, Caro JJ, Cramer J, Daley WL, Gurwitz J, Hollenberg NK: Recommendations for evaluating compliance and persistence with hypertension therapy using retrospective data. Hypertension 2006;47:1039-1048.

14 Vollmer WM, Xu M, Feldstein A, Smith D, Waterbury A, Rand C: Comparison of pharmacy-based measures of medication adherence. BMC Health Serv Res 2012;12:155.

15 Munck LK, Hansen KR, Mølbak AG, Balle H, Kongsgren S: The use of shared medication record as part of medication reconciliation at hospital admission is feasible. Dan Med J 2014;61:A4817.

16 Steiner JF, Koepsell TD, Fihn SD, Inui TS: A general method of compliance assessment using centralized pharmacy records. Description and validation. Med Care 1988;26:814-823. 
Hedegaard et al.: Multifaceted Intervention Including Motivational Interviewing to Support Medication Adherence after Stroke/TIA: A Randomized Trial

17 Morgado MP, Morgado SR, Mendes LC, Pereira LJ, Castelo-Branco M: Pharmacist interventions to enhance blood pressure control and adherence to antihypertensive therapy: review and meta-analysis. Am J Health Syst Pharm 2011;68:241-253.

-18 Jørgensen HS, Nakayama H, Reith J, Raaschou HO, Olsen TS: Acute stroke with atrial fibrillation. The Copenhagen Stroke Study. Stroke 1996;27:1765-1769.

19 Farmer KC: Methods for measuring and monitoring medication regimen adherence in clinical trials and clinical practice. Clin Ther 1999;21:1074-1090, discussion 1073.

20 Demonceau J, Ruppar T, Kristanto P, Hughes DA, Fargher E, Kardas P, De Geest S, Dobbels F, Lewek P, Urquhart J, Vrijens B: Identification and assessment of adherence-enhancing interventions in studies assessing medication adherence through electronically compiled drug dosing histories: a systematic literature review and meta-analysis. Drugs 2013;73:545-562.

21 Lager KE, Mistri AK, Khunti K, Haunton VJ, Sett AK, Wilson AD: Interventions for improving modifiable risk factor control in the secondary prevention of stroke. Cochrane Database Syst Rev 2014;5:CD009103.

22 McAlister FA, Majumdar SR, Padwal RS, Fradette M, Thompson A, Buck B, Dean N, Bakal JA, Tsuyuki R, Grover S, Shuaib A: Case management for blood pressure and lipid level control after minor stroke: PREVENTION randomized controlled trial. CMAJ 2014;186:577-584.

23 LaFleur J, Oderda GM: Methods to measure patient compliance with medication regimens. J Pain Palliat Care Pharmacother 2004;18:81-87.

24 O'Carroll RE, Chambers JA, Dennis M, Sudlow C, Johnston M: Improving adherence to medication in stroke survivors: a pilot randomised controlled trial. Ann Behav Med 2013;46:358-368.

25 Mackenzie G, Ireland S, Moore S, Heinz I, Johnson R, Oczkowski W, Sahlas D: Tailored interventions to improve hypertension management after stroke or TIA-phase II (TIMS II). Can J Neurosci Nurs 2013;35:27-34.

26 Flemming KD, Allison TG, Covalt JL, Herzig DE, Brown RD: Utility of a post-hospitalization stroke prevention program managed by nurses. Hosp Pract (1995) 2013;41:70-79.

27 Krska J, Rowe PH: Outcome measures: a sensitive approach. Int J Pharm Pract 2010;18:125-127.

28 Ho PM, Bryson CL, Rumsfeld JS: Medication adherence: its importance in cardiovascular outcomes. Circulation 2009; 119:3028-3035. 